\title{
El tratamiento del tabaquismo en alcohólicos: ¿miopía o negligencia?
}

\author{
Gemma Nieva; Antoni Gual \\ Unitat d' Alcohologia de la Generalitat. ICPP. Hospital Clínic. Barcelona. \\ Enviar correspondencia a: \\ gnieva@ico.scs.es
}

\section{RESUMEN}

Las drogodependencias más prevalentes en nuestra sociedad son el tabaquismo y el alcoholismo. En el caso de la población alcohólica, el consumo de tabaco está presente en un alto porcentaje de casos. Las repercusiones del consumo de ambas sustancias a la vez son devastadoras. Estudios que analizan la causa de muerte han identificado el tabaquismo como principal factor responsable de muerte en alcohólicos abstinentes. Sorprendentemente, poca ha sido la atención que ha merecido la dependencia tabáquica de estos pacientes por parte de los profesionales de los centros de drogodependencias de nuestro país.

Este artículo pretende evidenciar la importancia que juegan los profesionales para cambiar esta realidad y mejorar la calidad de vida de esta población. Tradicionalmente, la intervención en tabaquismo se aplaza tras varios años de abstinencia alcohólica, sin embargo los últimos hallazgos apuntan que tres meses después de la última ingesta, es tiempo suficiente para que el tratamiento del hábito tabáquico no aumente el riesgo de recaída alcohólica. Así mismo, hay tratamientos efectivos para facilitar el proceso de dejar de fumar en estos pacientes.

Palabras clave: alcohólicos fumadores, tabaco, alcohol, adicción.

\begin{abstract}
Smoking and alcohol drinking are the most common addictions in our society. In an alcohol-dependant population, tobacco use is present in a high percentage of cases. The health consequences of concurrent consumption are devastating. Research into mortality rates has identified tobacco use as the leading cause of death among abstinent alcoholics. Surprisingly, the tobacco dependence of these patients has not received the attention it merits from health professionals working in drug abuse centres in our country.

This article endeavours to show the importance of the role of health professionals in changing this situation and in improving the quality of life of these patients. Traditionally, treating tobacco use was delayed until after several years of alcohol abstinence. However, the latest evidence shows that a lapse of three months after the last drink is enough to commence smoking cessation intervention without jeopardizing alcohol abstinence. Moreover, there are effective treatments in facilitating smoking cessation in these patients.
\end{abstract}

Key words: smoker-alcoholics, smoking, alcohol, addiction.

\section{INTRODUCCIÓN}

$\mathbf{T}$ abaquismo y alcoholismo son las drogodependencias más extendidas en nuestra sociedad actual. Las substancias generadoras de estas adicciones comparten puntos en común. Ambas son legales, empeoran la calidad de vida de las personas que las padecen, son causa y agravantes de problemas de la salud y matan. La Organización Mundial de la Salud definió el cigarrillo como el único producto que usado, tal y como es indicado por fabricantes y autorizado por los gobiernos, mata. Sentencia ampliable al alcohol. Aunque en 2001 la mortalidad atribuible al tabaquismo descendió ligeramente por primera vez respecto a años anteriores en España, ésta ascendió a 54.233 muertes, $91 \%$ en varones ${ }^{1}$. El alcohol es responsable de casi 20.000 muertes más anualmen$t^{2}$. Tabaco y alcohol son la primera y tercera causa respectivamente de años de vida con discapacidades (OMS, 2004).

La asociación tabaco-alcohol en el consumo es muy frecuente. De hecho, entre el 80 y el $95 \%$ de alcohólicos fuman, lo que supone un porcentaje tres 
veces mayor que en la población general ${ }^{3}$. A la vez, los fumadores tienen un grado de dependencia del alcohol mayor ${ }^{4}$. Se estima que el alcoholismo es 10 veces más común en fumadores que en no fumadores $^{5}$.

Los efectos del consumo conjunto de alcohol y tabaco en la salud son devastadores. El riesgo de enfermedad y muerte es mayor que la suma de ambos riesgos. Y la población alcohólica es la primera víctima, a menudo sin tener la información del

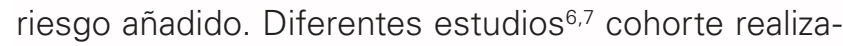
dos en el extranjero y también en el ámbito nacional, en nuestra realidad, demuestran que el tabaco es la causa principal de muerte en poblaciones tratadas por alcoholismo u otras drogodependencias no nicotínicas. Tras estos hallazgos, lo lógico seria abordar el tabaquismo de estos pacientes. La ciencia acumula conocimiento, y los profesionales debemos sacar provecho y adaptar nuestro modus operandi a la nueva situación. Pero esto no parece ser lo más frecuente, en este caso. En nuestro país, en muchos de los centros de drogodependencias se permite el consumo de tabaco, ello no merece otro calificativo que el de barbaridad. Entonces, ¿qué estamos dando a entender? Afortunadamente, hay esperanza para que esto cambie, y parece ser una prioridad del gobierno vigente que así sea. Claro está que no solamente se trata de legislar, sino de aplicar la normativa, y ahí los profesionales sanitarios, vamos a tener un papel crucial.

\section{CUANDO EL ABORDAJE DEL TABAQUISMO SE "OLVIDA"}

A estas alturas y con los datos que se barajan actualmente, nadie duda que la mejor decisión que puede tomar un fumador para su salud, es dejar de fumar. Igualmente, los sanitarios estamos de acuerdo en que si hay un problema de alcoholismo, la mejor determinación que puede tomar esta persona es abandonar el alcohol. Pero, ¿qué pasa cuando el paciente, además de tener problemas con el alcohol, es fumador? Pues, en la mayoría de los centros de nuestro país, cuando se aborda la dependencia del alcohol, se olvida el tabaco. Y, después del saber acumulado, ¿cómo es posible? Justamente por la alta prevalencia de fumadores en la población alcohólica o adicta a otras drogas, el tratamiento de la dependencia tabáquica es esencial también para estos pacientes. Es más, a veces es el propio paciente que ha dejado el alcohol pero sigue fumando, quién reclama una ayuda, una pista. Muchos de estos pacientes se sienten terriblemente dependientes y viven el tabaquismo como una esclavitud. Tienen la experiencia de haberse liberado de una cadena muy pesada, que era el alcoholismo, y demandan a voces un soporte para liberarse, esta vez, del tabaco. Actualmente, no informar a los alcohólicos de los riesgos añadidos del consumo conjunto de tabaco y alcohol, y no integrar en los protocolos de actuación de los centros de drogodependencias el abordaje del tabaquismo, puede calificarse de autentica negligencia.

Sin duda, los hábitos y actitudes de los profesionales de los centros de drogodependencias han influido en estas prácticas. Una proporción relevante de los terapeutas de estos centros son fumadores y muy pocos han integrado como parte de su rutina alguna acción preventiva referente al tabaco. Para cambiar esta realidad, los profesionales trabajadores de estos centros deben tener la suficiente formación para implementar tratamiento para dejar de fumar, que considere factores tanto físicos, como psicológicos y sociales, así como ofrecer tratamientos farmacológicos ceñidos a las necesidades personales de cada paciente. La regulación de todos los espacios cerrados como centros sin humos, es vital para desnormalizar el uso del tabaco. Y los centros de drogodependencias no deben ser una excepción.

Los tratamientos para la deshabituación del alcohol son largos, a menudo duran varios años. Durante este tiempo los pacientes acuden a terapias, de frecuencias variables, a veces mensuales, pero a veces incluso semanales. A lo largo de este tiempo, se establece una relación entre el equipo terapéutico y paciente, que debe aprovecharse para hablar del tabaco e incidir en el camino hacia el cese. De lo contrario, no estamos ejerciendo nuestras responsabilidades respecto a la salud de los pacientes.

\section{CUANDO INICIAR EL ABORDAJE DEL TABAQUISMO}

Hasta hace poco tiempo se aplazaba el tratamiento del tabaquismo hasta que la abstinencia alcohólica fuera de años, a pesar de la ausencia de evidencia científica que lo aconsejara. En los últimos años, se ha incluido el abordaje del tabaquismo en pacientes alcohólicos especialmente en algunos centros de Estados Unidos, pero quedan por definir cuáles son las estrategias óptimas para esta población. En los estudios realizados, un mensaje queda claro: tener historia de alcoholismo no impide poder dejar de fumar ${ }^{8}$ sin que ello signifique tener más recaídas alcohólicas ${ }^{9,10}$.

Una de las cuestiones claves que quedan por resolver es el momento de la intervención tabáquica. Algunos estudios están evaluando en la actualidad cuál es el mejor momento para ayudar a dejar de fumar. Existe poca evidencia científica al respecto. 
Uno de los primeros estudios publicados contestando esta cuestión, afirma que en población alcohólica con abstinencia mayor de tres meses, la recaída etílica no se relaciona con el status de fumador, y tampoco hay relación entre el tiempo de abstinencia alcohólica y el éxito en dejar de fumar ${ }^{11}$. Cabe deducir que los tres meses de abstinencia alcohólica son suficientes para evitar recaídas, pero es necesaria la realización de estudios adicionales que concreten el mejor momento para intervenir en tabaquismo para aportar mayor solidez científica a los datos publicados.

\section{TRATAMIENTO}

El abordaje del tabaquismo no se puede reducir únicamente a temas farmacológicos, y por tanto debe tenerse en cuenta lo psicológico y social. En ese sentido, todos los esfuerzos para aumentar la importancia y la autoeficacia para abandonar el tabaco, serán logros de gran utilidad.

En el ámbito farmacológico, disponemos de diferentes herramientas para ofrecer a los fumadores con historia de alcoholismo. Los parches de nicotina son eficaces para evitar el síndrome de abstinencia tabáquico de esta población ${ }^{12}$. También el bupropion, una medicación no nicotínica, ha demostrado efectividad para alcohólicos abstinentes ${ }^{13}$.

Nuevos fármacos están siendo evaluados con el mismo fin. Uno de ellos, el topiramato, parece ser efectivo para el tratamiento de ambas sustancias, presumiblemente porque actúa modulando la función dopaminérgica a nivel cortico-mesolímbico ${ }^{14}$. Nuevamente, más investigaciones son necesarias para dar firmeza a estos hallazgos.

\section{CONCLUSIONES}

Los profesionales sanitarios somos clave para disminuir la mortalidad evitable debida al consumo de tabaco y alcohol. Dejar de fumar debe ser tan importante para pacientes sin historia alcohólica o de otras drogadicciones, como para pacientes con ella. Estos enfermos obtienen beneficios en todos los ámbitos personales cuando dejan de fumar. Además pueden conseguirlo igual que los demás. A pesar de ello, una gran parte del sector especialista en drogodependencias se resiste a incorporar el tratamiento del tabaquismo en estos pacientes, ¿se merece ello el calificativo de negligencia? Como promotores de salud que somos, no podemos renunciar a nuestras responsabilidades y debemos, sin excepción alguna, integrar en nuestro trabajo la desnormalización de la droga más institucionalizada que existe: el tabaco.

\section{REFERENCIAS}

1. Banegas JR, Díez Gañán L, González Enríquez J, Villar Alvarez F, Rodríguez-Artalejo F. La mortalidad atribuible al tabaquismo comienza a descender en España. Med Clin 2005; 124: 769-71.

2. Portella E, Ridao M, Carrillo E, Ribas E, Ribó C, Salvat M. El alcohol y su abuso: impacto socioeconómico. Madrid:Ed. Médica Panamericana; 1998.

3. Alcohol Alert, 39, National Institute on Alcohol Abuse and Alcoholism; 1998.

4. Daeppen JB, Smith TL, Danko GP, Gordon L, Landi NA, Nurnberger JI Jr, Bucholz KK, Raimo E, Schuckit MA. Clinical correlates of cigarette smoking and nicotine dependence in alcohol-dependent men and women. The Collaborative Study Group on the Genetics of Alcoholism. Alcohol Alcohol. 2000; 35: 171-5.

5. DiFranza JR, Guerrera MP. Alcoholism and smoking [resumen]. J Stud Alcohol 1990; 51:130-5.

6. Hurt RD, Offord KP, Croghan IT, Gomez-Dahl L, Kottke TE, Morse RM, Melton LJ 3rd. Mortality following inpatient addictions treatment. Role of tobacco use in a community-based cohort. JAMA 1996; 275: 10971103.

7. Gual A, Lligoña A, Costa S, Segura L, Colom J. El tratamiento del alcoholismo y su impacto a largo plaxo. Resultados a 10 años de un estudio longitudinal prospectivo de 850 alcohólicos tratados en la red pública de Cataluña. Med Clin 2004; 124: 769-71.

8. Hughes JR, Callas PW. Past alcohol problems do not predict worse smoking cessation outcomes. Drug Alcohol Depend 2003; 10;71: 269-73.

9. Friend KB, Pagano ME. Smoking cessation and alcohol consumption in indivuduals in treatment for alcohol use disorders. J Addict Dis 2005; 24: 61-75.

10. Bobo JK, Mcllvain HE, Lando HA, Walker RD, LeedKelly A. Effect of smoking cessation counseling on recovery from alcoholism: findings from a randomized community tintervention trial. Addiction 1998; 93: 877-87.

11. Martin JE, Clafas KJ, PattenCA, Polarek M, Hofstetter CR, Noto J Beach D. Prospective evaluation of three smoking interventions in 205 recovering alcoholics: one year resluts of Project SCRAP-Tobacco. J Consult Clin Psychol 1997; 65:190-4.

12. Hughes JR, Novy P, Hatsukami DK, Jensen J, Callas PW. Efficacy of nicotine patch in smokers with a 
history of alcoholism. Alcohol Clin Exp Res 2003; 27: 946-54.

13. Hayford KE, PattenCA, RummansTA, Schroeder DR, Offord KP, Croghan IT et al. Efficacy of bupropion for smoking cessation in smokers with a former history of major depression or alcoholism. Br J Psychiatry 1999; 174: 173-8.

14. Johnson BA. Topiramate-induced reuromodulation of cortico-mesolimbic dopamine function: A new vista for the treatment of comorbid alcohol and nicotine dependence? Addictive Behaviors 2004; 29: 1465-79. 